\title{
METALLICITY EFFECTS IN MASSIVE STAR EVOLUTION AND NUMBER FREQUENCIES OF WR STARS IN GALAXIES
}

\author{
André Maeder \\ Geneva Observatory \\ CH-1290 Sauverny, Switzerland
}

\begin{abstract}
The results of new grids of models of massive stars with metallicities $\mathrm{Z}=0.002,0.005$, 0.020 and 0.040 and mass loss rates depending on $\mathrm{Z}$ are shown. When integrated over the mass spectrum, the models enable us to predict number ratios, such as WR/O, WC/WN, WNE/WR, $\mathrm{WNL} / \mathrm{WR}, \mathrm{WCE} / \mathrm{WR}, \mathrm{WCL} / \mathrm{WR}, \mathrm{WO} / \mathrm{WR}$ as a function of $\mathrm{Z}$ in galaxies.

Comparisons between models and observations in galaxies are made and show, as was suggested by Maeder, Lequeux and Azzopardi (1980), that the effects of metallicity on the mass loss rates are the prime agent responsible for the different distributions of massive stars in galaxies.
\end{abstract}

\section{INTRODUCTION}

The changes of the WR populations in galaxies with active star formation were first discovered in the pioneer work by Smith $(1968,1973,1982)$. Further observational studies, particularly in the Magellanic Clouds by Breysacher (1981) and Azzopardi and Breysacher (1985) have confirmed the differences in the WR populations. Maeder, Lequeux and Azzopardi (noted MLA, 1980) have proposed an explanation of the observed differences in the number frequency of WR stars by a connection between the local metallicity $\mathrm{Z}$ and mass loss. At high $\mathrm{Z}$ (inner galactic locations), gas opacities are larger in the outer stellar layers, more momentum is therefore transferred by radiation pressure effects, mass loss by stellar winds in massive $\mathrm{O}$ stars is more intense and thus more bare cores are formed.

The proposition by MLA was criticised by Bertelli and Chiosi (1981) who claimed that the galactic gradient in WR stars simply reflects the gradient in $\mathrm{O}$ stars. Similar claims against metallicity effects were made by Armandroff and Massey (1985) and by Conti et al. (1983a). However, Meylan and Maeder (1983) showed that the galactic gradient of WR star numbers is steeper than the gradient in $O$ stars. Thus the WR distribution in the Galaxy is not just a reflection of the $\mathrm{O}$-star distribution and the WR gradient cannot be explained only by a change in the IMF.

Up to now, the interpretation of the observed trends have remained essentially qualitative. The aim of this work is to perform a quantitative approach on the basis of new grids of models of massive stars at various metallicities. 


\section{SUMMARY ON MODEL PHYSICS}

The models used here to derive the theoretical number frequencies of WR stars are the new grids of evolutionary models of massive stars at various metallicities $Z=0.002,0.005$, 0.020 , and 0.040 (cf. Maeder, 1990a). These models use up-to-date nuclear cross-sections and new opacity tables made by G. Schaller at Geneva Observatory from the Los Alamos Opacity Programme. Proper account is given in the opacity tables to the fact that the $\mathrm{O} / \mathrm{Fe}$ and $\alpha$-nuclei/Fe abundance ratios are larger than solar at low $\mathrm{Z}$. The initial model abundances have also been modified accordingly.

The mass loss rates for Pop. I stars with initial $\mathrm{Z}=0.020$ (except the WR stars) are based on recent data analyses by de Jager et al. (1988). At $Z$ values different from $Z=0.020$, the mass loss rates $\dot{M}$ have been scaled according to $\dot{M} \sim Z^{\xi}$. An exponent $\xi=0.5$ has been taken, as indicated by the stellar wind models by Kudritzki et al. (1987).

For WR stars, we no longer use the average observed mass loss rates, as we and others generally did in the past. As shown by Schmutz et al. (1989), this was leading to theoretical WR luminosities that were much higher than those observed. There were several indications in favour of $\dot{M}$-rates for WNE and WC stars depending on the actual masses of WR stars (cf. Abbott et al., 1986; St-Louis et al., 1988; Langer, 1989; Smith and Maeder, 1989). Langer's relation has been used here.

It has repeatedly been suggested (cf. Maeder and Meynet, 1989) that the models should also include the core-overshooting effect. Also, the $\mathrm{T}_{\text {eff-values of }} \mathrm{WR}$ stars have been corrected for the optical thickness of the wind, supposedly due mainly to electron scattering. Due to the $\mathrm{T}_{\text {eff }}$ correction, the WNE and WC stars follow unique well-defined tracks in the HR diagram, independently of their initial stellar masses. This can be seen, for example, in Fig. 3. These tracks are explained by the fact that as their mass and luminosity decline, the $\dot{M}$-rates and the amplitude of the $\mathrm{T}_{\text {eff }}$ correction are reduced, thus the WR stars move downwards and bluewards in the HR diagram.

\section{MODEL RESULTS CONCERNING WR STARS}

Surface abundances play a key role in the identification of models with WR subtypes. As is known, the observed abundances of WNL, WNE, WC and WO stars are consistent with the exposition of nuclearly processed materials. The correspondence applied between WR subtypes and surface abundances is discussed in the detailed paper (Mader, 1990b) on WR distributions in galaxies.

Fig. 1 gives an overview of the lifetimes $t_{W R}$ in the WR stage as a function of mass and $Z$. The clear trend - easily understandable - is an increase of $t_{W R}$ with initial mass and $\mathrm{Z}$. Also we notice that the minimum mass for WR formation is lower at higher $\mathrm{Z}$. Similar graphs can be established for the lifetimes in the WN-late, WN-early, WC-late and WC-early phases. 


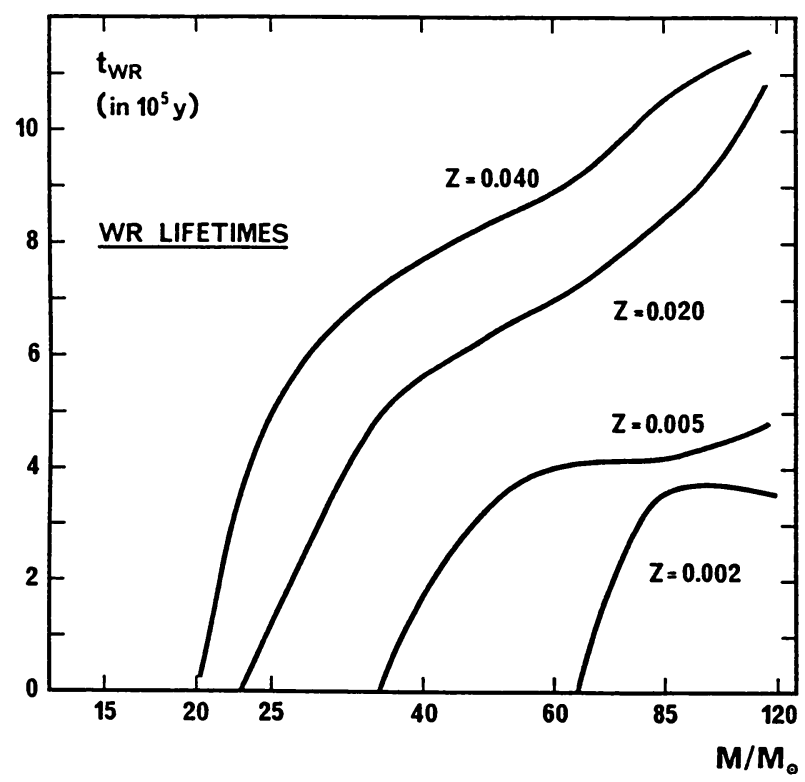

Figure 1 Lifetimes as Wolf-Rayet stars as a function of metallicity and initial stellar masses.

The lifetimes in the various phases can be used to derive relative number frequencies WR/O, WC/WR, WC/WN. Assumptions on the star formation rate (SFR) and the initial mass function (IMF) have of course to be made. For a galaxy or a large galactic ring, the asumption of a constant SFR over the last few $10^{7} \mathrm{y}$ is a reasonable one. (For a single HII region, this would not be acceptable and aging effects are likely to intervene). For the IMF, two forms $\mathrm{dN} / \mathrm{dM}=\mathrm{AM}^{-(1+x)}$ with $\mathrm{x}=1.35$ and 1.7 are considered. Table 1 shows the results for the number ratios $\mathrm{WR} / \mathrm{O}, \mathrm{WC} / \mathrm{WR}$ and $\mathrm{WC} / \mathrm{WN}$ at various metallicities. Two cases for the lower $\mathrm{T}_{\text {eff }}$ limit of $\mathrm{O}$ stars are considered, one at $\log \mathrm{T}_{\text {eff }}=4.52$ and the other at 4.53 .

The results of Table 1 can be compared with the observed number ratios in galaxies and galactic rings in the Milky Way. The main source references for the observations are Smith (1988), Arnault et al. (1989). For the Milky Way, both the data from van der Hucht et al. (1988) and Conti and Vacca (1990, squares in Fig. 2) are considered. As an example, Fig. 2 shows the comparison between the observed and theoretical WC/WN number ratios respectively. On the whole, the agreement is excellent, although there are some sizeable differences between the two sources of galactic data. Fig. 2 gives powerful support to the evolutionary models and to the idea by MLA (1980) that metallicity Z, through its effects on the mass loss rates $\dot{M}$ of $\mathrm{O}$ stars and supergiants, is responsible for the enormous differences of the WR populations in galaxies with active star formation. 
TABLE 1. Theoretical number ratios for Wolf-Rayet stars as a function of metallicity $\mathrm{Z}$, for 2 slopes $x$ of the IMF.

\begin{tabular}{|c||c|c|c|c|}
\hline $\mathrm{Z}$ & $\begin{array}{c}\mathrm{WR} / 0 \\
\log \mathrm{T}>4.53\end{array}$ & $\begin{array}{c}\mathrm{WR} / 0 \\
\log \mathrm{T}>4.52\end{array}$ & $\mathrm{WC} / \mathrm{WR}$ & $\mathrm{WC} / \mathrm{WN}$ \\
\hline$\frac{\mathrm{x}=1.35}{0.002}$ & 0.0034 & 0.0032 & 0.057 & 0.061 \\
0.005 & 0.0185 & 0.0182 & 0.192 & 0.237 \\
0.020 & 0.0790 & 0.0752 & 0.640 & 1.784 \\
0.040 & 0.1628 & 0.1557 & 0.736 & 2.784 \\
$\frac{\mathrm{x}=1.70}{0.002}$ & & & & \\
0.005 & 0.0021 & 0.0019 & 0.058 & 0.061 \\
0.020 & 0.0135 & 0.0133 & 0.170 & 0.204 \\
0.040 & 0.0654 & 0.0616 & 0.634 & 1.732 \\
\hline
\end{tabular}

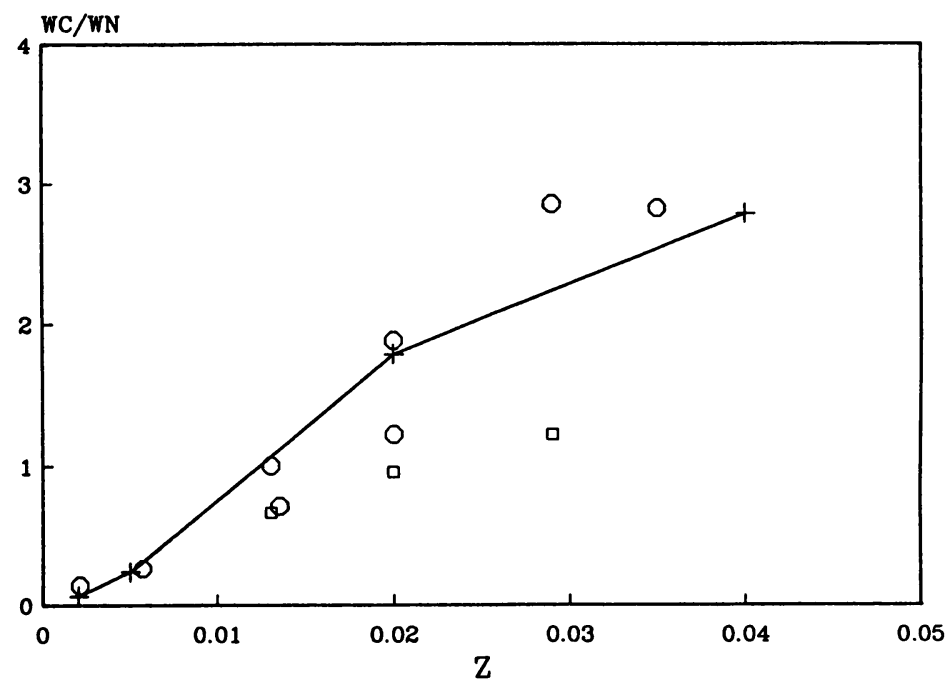

Fig. 2: Comparison of observed (circles, squares) and theoretical (line) number ratios WC/WN of WC stars to WN stars as a function of initial metallicity.

The relative number of WN stars with and without hydrogen, identified with WNL and WNE stars respectively, are also derived ( $\mathrm{L}$ for late, $\mathrm{E}$ for early). The WNL phases generally last much longer for large initial masses, with little dependence on $\mathrm{Z}$. On the other hand, the WNE phases are longer for lower initial masses, also being shorter at $\mathrm{Z}=0.04$ than at $\mathrm{Z}=0.02$. The comparisons with observations of WNL and WNE stars in galaxies show agreement for $\mathrm{Z} \geq 0.02$. At lower $\mathrm{Z}$, there are more WNE stars than predicted and we conclude that a large fraction of the existing WR stars probably results from binary evolution and that they are mostly WNE stars. 
The relative numbers of WC stars with various subtypes are also analysed quantitatively. WC models are classified according to the $(\mathrm{C}+\mathrm{O}) / \mathrm{He}$ number ratios (cf. Smith and Hummer, 1988; Smith and Maeder, 1990):

$\begin{array}{llll}\text { WC9 } & (\mathrm{C}+\mathrm{O}) / \mathrm{He}=0.03 \text { to } 0.06 & \text { WC5 } & (\mathrm{C}+\mathrm{O}) / \mathrm{He}=0.55 \\ \text { WC8 } & (\mathrm{C}+\mathrm{O}) / \mathrm{He}=0.1 & \text { WC4 } & (\mathrm{C}+\mathrm{O}) / \mathrm{He}=0.7 \text { to } 1.0 \\ \text { WC7 } & (\mathrm{C}+\mathrm{O}) / \mathrm{He}=0.2 & \text { WO } & (\mathrm{C}+\mathrm{O}) / \mathrm{He} \text { greater than } 1.0 \\ \text { WC6 } & (\mathrm{C}+\mathrm{O}) / \mathrm{He}=0.3 & & \end{array}$

The models show that the entry points and lifetimes in the sequence WC9 $\rightarrow$ WC4, WO are extremely Z- and mass-dependent. The entry into WC9 subtype only occurs for high initial $\mathrm{Z}$ and mass (i.e. $\mathrm{Z}>0.02$ and $\mathrm{M} \geq 50 \mathrm{M}_{\odot}$, cf. Fig. 3). Lower initial $\mathrm{M}$ and $\mathrm{Z}$ lead to entry at earlier WC subtypes, with a shorter overall WC phase. After integration of the lifetimes over the mass spectrum, we notice that at high initial $\mathrm{Z}$, late and intermediate WC subtypes are favoured, while at lower Z, only early WC and WO stars are found and in much lower frequencies. The theoretical number ratios of late, early WC and WO stars compare quite well with observed number ratios in galaxies of the Local Group. Space and time requirements preclude these results to be shown in detail here, and we therefore refer the reader to the works by Smith and Maeder (1990) and Maeder (1990b).

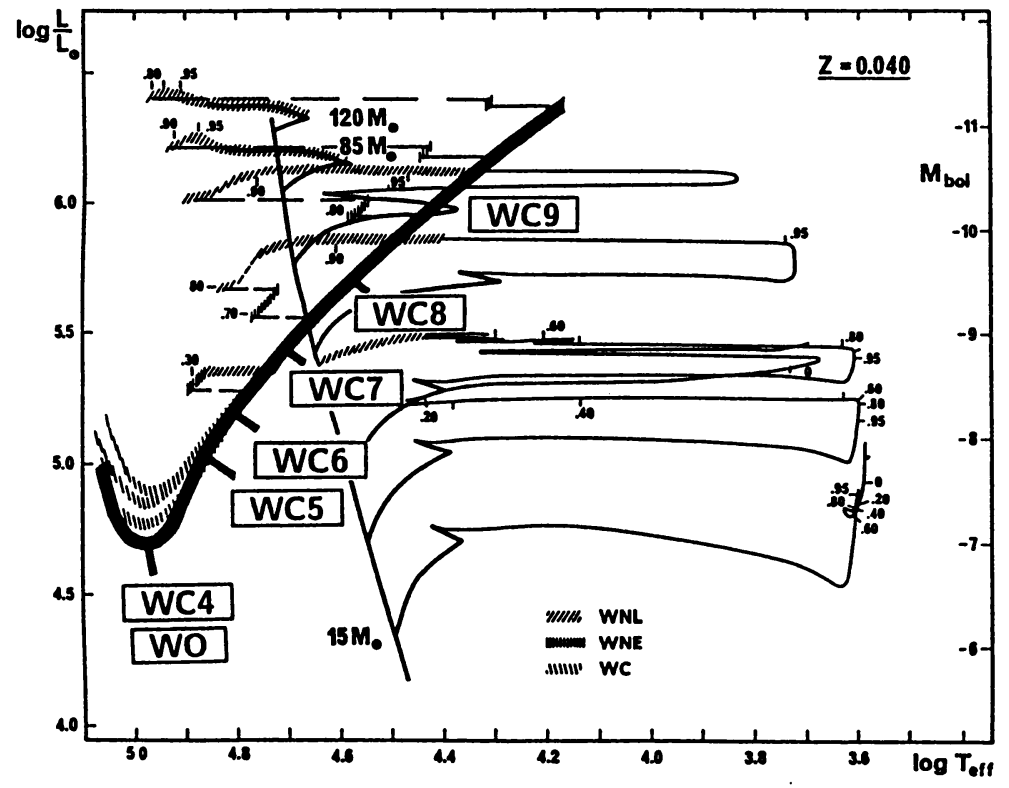

Fig. 3: Evolutionary tracks in the $H R$ diagram for models with $Z=0.040$. The average intervals occupied by the existing WC subtypes and WO stars are indicated along the thick line, on the basis of the $(\mathrm{C}+\mathrm{O}) / \mathrm{He}$ number ratios. 


\section{REFERENCES}

Abbott D., Bieging J.H., Churchwell E., Torres A.V.: 1986, Astrophys. J. 303, 239

Armandroff T.E., Massey P.: 1985, Astrophys. J. 291, 685

Arnault P., Kunth D., Schild H.: 1989, Astron. Astrophys. 224, 73

Azzopardi M., Breysacher J.: 1985, Astron. Astrophys. 149, 213

Bertelli G., Chiosi C.: 1981, in The most massive stars, Ed. S. D'Odorico et al., ESO, Garching, p. 211

Breysacher J.: 1981, Astron. Astrophys. Suppl. Ser. 43, 203

Conti P.S., Garmany C.D., de Loore C., Vanbeveren D.: 1983a, Astrophys. J. 274, 302

Conti P.S., Vacca B., 1990: Astrophys. J. in press

van der Hucht K.A., Hidayat B., Admiranto A.G., Supelli K.R., Doom C.: 1988, Astron. Astrophys. 199, 217

de Jager C., Nieuwenhuijzen H., van der Hucht K.A.: 1988, Astron. Astrophys. Suppl. Ser. 72, 259

Kudritzki R.P., Pauldrach A., Puls J.: 1987, Astron. Astrophys. 173, 293

Langer N.: 1989, Astron. Astrophys. 220, 135

Maeder A.: 1990a, Astron. Astrophys. Suppl. Ser. 84, 139

Maeder A.: 1990b, Astron. Astrophys. in press

Maeder A., Lequeux J., Azzopardi M.: 1980, Astron. Astrophys. 90, L17 (MLA)

Maeder A., Meynet G.: 1989, Astron. Astrophys. 210, 155

Meylan G., Maeder A.: 1983, Astron. Astrophys. 124, 84

Schmutz W., Hamann W.-R., Wessolowski K.: 1989, Astron. Astrophys. 210, 236

Smith L.F.: 1968, M.N.R.A.S. 141, 317

Smith L.F.: 1973, IAU Symp. 49, 15

Smith L.F.: 1982, IAU Symp. 99, 597

Smith L.F.: 1988, Astrophys. J. 327, 128

Smith L.F., Hummer, D.G.: 1988, M.N.R.A.S. 230, 511

Smith L.F., Maeder A.: 1990, Astron. Astrophys. in press

St. Louis N., Moffat A.F.J., Drissen L., Bastien P., Robert C.: 1988, Astrophys. J. 330, 286

\section{DISCUSSION}

Moffat: This work is very impressive and answers many questions. But one problem is with the masses of WR stars for different ambient $Z$. Contrary to your predictions, WR star masses in the LMC and SMC are the same as the masses of their galactic counterparts, despite the large range in $Z$ ( $c f$. poster on WR masses from polarization data at this symposium). Note that the binary frequency, e.g., of WNL stars is identical in the Galaxy and the LMC (Moffat, 1989) while in the SMC the numbers are too small to say, although certainly not all WR stars in the SMC are binaries. Hence, duplicity cannot play a strong role in WR evolution.

Maeder: I am not sure that the distributions of WR star masses in the Galaxy, the LMC and in the SMC are known sufficiently well to allow us to claim for differences or similarities between them. On the contrary, I do think from available data that at low $Z$, WR formation mainly occurs from binary evolution. 
Lortet: About WC/WN ratios: there are large error bars on observational counts (see section WR inventory). There are huge selection effects, favouring the easy detection of strong lined stars. Even in the LMC, Morgan and Good explain their survey only covers stars with $E W>30 \AA$.

Maeder: You are the observer, and you never put the error bars on your results, thus I look forward to see them in future.

Massey: You compare your model predictions to the number of WR/O stars and the ratio $\mathrm{WC} / \mathrm{WR}$ stars in a number of local group galaxies. The number of $\mathrm{O}$ stars (e.g., massive progenitors) in these galaxies is not known to even astronomical accuracy. I also note the poster paper by Armandroff and Massey: the ratio WC/WN is at most 0.9 in M31, based on the spectroscopically confirmed WR stars. In fact, it appears that the WC/WN ratio's in M33 and M31 are quite similar. Metallicity cannot be the only parameter controlling this ratio.

Maeder: Apart from metallicity, the IMF and the star formation rate may also play a role. For example, in a small starburst region the WR/O and WC/WR ratio's may change as the starburst region is aging. But, I doubt that this applies to a galaxy as M31 or M33, where we see the results of star formation rates averaged over very large regions.

Sreenivasan: I have a fundamental concern with your models. It is hard to understand on any physical basis why all massive stars have to overshoot by the same amount $d=0.25$ and why massive stars obey a mass loss rate relationship that depends upon the mass as well as other properties but suddenly switch to obeying a relationship that depends only upon the mass when their surface hydrogen composition drops to 0.2 . We must remember that correlations do not imply causal relationships and that statistical fits to observed data may later not be "unfolded" to suit individual cases. This conclusion is evidenced by the fact that your observational input to theoretical modeling does not predict other observed fits!

Maeder: There are no reliable wind models for stars outside the MS band and we have to base the models on the observed mass loss rates. If I remember correctly, are you not also using some kind of parametrisation in your own models?

Walborn: What theoretical parameter determines your linear sequence of WC subtypes in the HR diagram, and is it well established observationally?

Maeder: The parameters determining the sequence of WC subtypes are the $(C+O) / H e$ abundance ratios. This is based on the determinations of $(\mathrm{C} / \mathrm{He})$ ratios by Smith and Hummer (1988) and on the discussion and calibration established by Smith and Maeder (1990).

Vanbeveren: It must be realised that an uncertainty of a factor 2 in the observed $\dot{M}$ values produces a very large uncertainty in the evolutionary results. We really need $\dot{M}$ values with accuracies better than a factor 2 . Maybe for the future it would be better, instead of observing many stars only a few times, if one would observe only a few stars many times. Maeder: The very high sensitivity of evolution on the mass loss rates is precisely what was emphasised over the last ten years. Thus, as you are mentioning, within the range of uncertainties of the mass loss rates, one may have a certain variety of evolutionary tracks at a given inital mass.

Humphreys: Have you considered the role of other effects such as population statistics on the members of WR stars of different types? E.g., we know that the luminosity of the most luminous star depends on the luminosity of the parent galaxy; the smaller less luminous systems have fewer of the most massive stars. These galaxies also tend to have the lowest metallicity. Is the apparent dependence on metallicity due at least in part to population statistics?

Maeder: R. Schild and myself (1984) made a population synthesis to study this effect. 


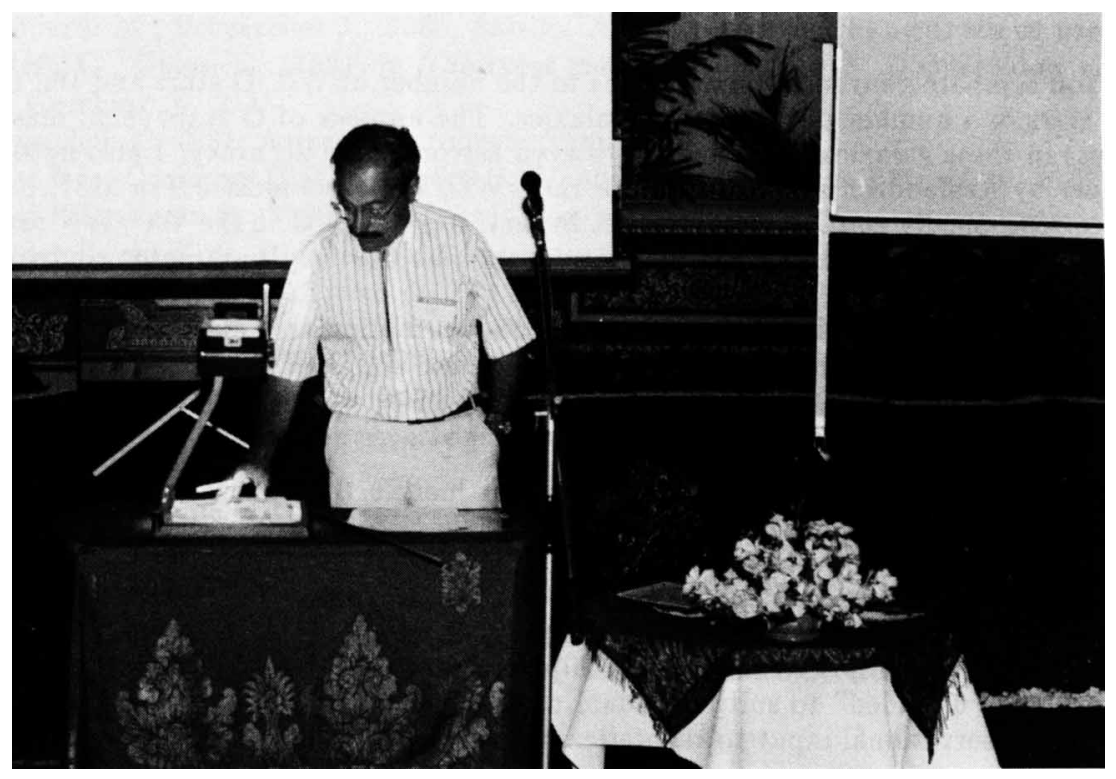

André Maeder 